\title{
Adoption complications
}

\begin{abstract}
"Try to find an adoptive home for an animal that was used on a research project." This was one of the guiding principles for the staff of the laboratory animal facility at Great Eastern University. Therefore, it came as no surprise to anybody that Dr. Linda Roman spayed Fifi, a healthy 2-year old cat that was no longer being used on a research project and gave her to a research technician who was fond of the animal. The technician had previously completed a University-approved form, which stated that the animal would be kept only as an indoor-housed pet and would receive any needed veterinary care from a private practitioner. Additionally, Max Levine, the Principal Investigator (PI), had previously signed a document allowing the technician to adopt the cat. During Levine's animal behavior study, Fifi did not experience any
\end{abstract}

pain or distress, and, therefore, the IACUC had placed her in USDA Annual Report Category C (no pain or distress).

When the paperwork reached the IACUC office, the IACUC administrator began to think about what had transpired. It was true that the policy of adopting out a research animal was not a new one, but now she was having second thoughts about the process. Every animal had to be covered by an IACUC protocol, but Fifi seemed to fall between the cracks. Was she still Levine's cat during and just after being spayed? If so, should the IACUC have placed her in USDA Category $\mathrm{D}$ (alleviated pain or distress)? On the other hand, because the study was finished, should the IACUC consider the spaying surgery a veterinary clinical procedure? Even with that situation, should the IACUC cover her with a protocol until she went home with the tech- nician? Levine didn't want any part of the discussion. He said he was just trying to be helpful and didn't want to become involved in any of the politics. Roman said the same thing. Unfortunately, the IACUC didn't know what to do and briefly considered contacting both the Office of Laboratory Animal Welfare (OLAW) and US Department of Agriculture (USDA) Animal Care Division. The Committee decided to use that option as a last resort action because it didn't want to be juggling opinions from two federal oversight agencies.

How would you approach this situation? Under the current circumstances, should Fifi remain in USDA Category $\mathrm{C}$ or be moved into Category D? Did she need to be covered by an IACUC protocol once the study ended? Should the IACUC change its adoption procedure?

\section{RESPONSE}

\section{USDA pain categories do not apply}

\section{Eva B. Ryden, PhD, DVM, DACLAM \& Kelly M. Conway, BS, LATG}

Neither the Animal Welfare Act (AWA $)^{1}$ nor the Public Health Service Policy on Humane Care and Use of Laboratory Animals (PHS Policy $)^{2}$ includes any policies addressing the adoption of research animals. The AWA $(\$ 2.35 . c .1,2,3$. ) record-keeping requirements state that "...every research facility transporting, selling or otherwise disposing of any live dog or cat to another person shall make and maintain records...etc." OLAW Policies and "Dear Colleague" letters do not address the issue of adoption. USDA Animal Care Policy \#11 (Painful Procedures ${ }^{3}$ ) addresses itself to the IACUC, and Policy \# 12 (Written Narrative for Alternatives to
Painful Procedures ${ }^{4}$ ) addresses itself to PIs, indicating that consideration of assignment to a USDA pain category applies primarily or exclusively to research animals.

Therefore, as implied in The IACUC Handbook $^{4}$, it is permissible for Institutional Policies in the spirit of the AWA and PHS Policy to be promulgated and followed. With respect to Fifi's case, the questions must then reflect the IACUC's best judgment for the animal and the institution. The IACUC may choose to institute the following policy: "Providing that the animals (considered for adoption) are healthy and have not been disfigured or disabled by the research, adoption of animals that make suitable pets is appropriate 5 ." In fact, nobody at Great Eastern is disputing this plan. Levine, the PI, had "previously signed a document allowing the technician to adopt the cat." Hence, in our judgment, when Fifi was spayed, she no longer belonged to Levine. Because she had not been transferred to another IACUC protocol but had by the completion of that paperwork become a pet, the USDA Policy \# 11 no longer covered her. Therefore, it is not necessary to assign a USDA Pain Category to Fifi for her spay surgery.

One might question the advisability of Roman's spaying Fifi at Great Eastern. It is doubtful whether this can qualify as a "veterinary clinical procedure" in the sense intended by the AWA ( $\$ 2.31$.d.ix.B.), that is, "required as a routine veterinary procedure or to protect the health or wellbeing of the animal as determined by the Attending Veterinarian." This paragraph in fact refers to multiple major survival surgeries and covers animals on IACUC protocols. Because spaying Fifi is not necessary for her health or to be adopted and go home with her new owner, it is probably advisable to require, as part of the adoption agreement, that her new owner sign a statement that Fifi will be spayed and will not be allowed to breed. Moreover, the research technician adopting Fifi has already signed a statement that "the ani- 